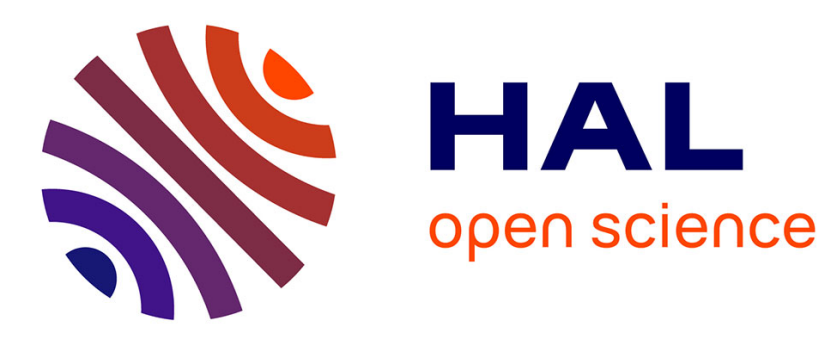

\title{
Long Range Energy Transfer in Self-Assembled Stacks of Semiconducting Nanoplatelets
}

Jiawen Liu, Lilian Guillemeney, Benjamin Abecassis, Laurent Coolen

\section{To cite this version:}

Jiawen Liu, Lilian Guillemeney, Benjamin Abecassis, Laurent Coolen. Long Range Energy Transfer in Self-Assembled Stacks of Semiconducting Nanoplatelets. Nano Letters, 2020, 10.1021/acs.nanolett.0c00376 . hal-02565031

\section{HAL Id: hal-02565031 \\ https://hal.science/hal-02565031}

Submitted on 6 May 2020

HAL is a multi-disciplinary open access archive for the deposit and dissemination of scientific research documents, whether they are published or not. The documents may come from teaching and research institutions in France or abroad, or from public or private research centers.
L'archive ouverte pluridisciplinaire HAL, est destinée au dépôt et à la diffusion de documents scientifiques de niveau recherche, publiés ou non, émanant des établissements d'enseignement et de recherche français ou étrangers, des laboratoires publics ou privés. 


\title{
Long range energy transfer in self-assembled stacks of semi-conducting nanoplatelets
}

\author{
Jiawen Liu ${ }^{1}$, Lilian Guillemeney ${ }^{2}$, Benjamin Abécassis ${ }^{2}$ and Laurent Coolen ${ }^{1, *}$ \\ ${ }^{1}$ Sorbonne Université, CNRS, Institut de NanoSciences de Paris, INSP, F-75005 Paris, France \\ ${ }^{2}$ Univ Lyon, CNRS, École Normale Supérieure de Lyon, Laboratoire de Chimie UMR 5182, 46 allée d'Italie, \\ F-69007 Lyon, France
}

\begin{abstract}
Fluorescent emitters like ions, dye molecules or semiconductor nanoparticles are widely used in opto-electronic devices, usually within densely-packed layers. Their luminescence properties can then be very different from when they are isolated, because of short-range interparticle interactions such as Förster resonant energy transfer (FRET). Understanding these interactions is crucial to mitigate FRET-related losses and could also lead to new energy transfer strategies. Exciton migration by FRET hopping between consecutive neighbor fluorophores has been evidenced in various systems but was generally limited to distances of tens of nanometers and involved only a few emitters. Here we image selfassembled linear chains of CdSe nanoplatelets (colloidal quantum wells) and demonstrate exciton migration over 500-nm distances, corresponding to FRET hopping over 90 platelets. By comparing a diffusion-equation model to our experimental data, we measure a (1.5 ps) $)^{-1}$ FRET rate, much faster than all decay mechanisms, so that strong FRET-mediated collective photophysical effects can be expected.
\end{abstract}

Keywords : self-assembly ; nanoplatelets ; FRET ; exciton transfer ; fluorescence microscopy

FRET is a non-radiative energy transfer mechanism by electromagnetic dipole-dipole coupling ${ }^{1}$. FRET energy migration plays a key role in photosynthesis by funneling photogenerated excitons from the light harvesting centers to the reaction center ${ }^{2}$. Systems of dyes attached to DNA scaffolds have been engineered in order to create FRET-based bioinspired 30-nm photonic wires ${ }^{3}$ and logic gates ${ }^{4}$. In opto-electronics, FRET must be understood as it may be detrimental by funneling excitonic energy to quenching sites, but it can also favor efficient charge collection in photovoltaic cells and lead to new FRET-enabled excitonic devices 5 .

With this in mind, exciton migration has been explored in various systems. In molecular or polymer systems, migration by incoherent FRET hopping was generally demonstrated over a few tens of nanometers ${ }^{6-8}$ (while distances of hundreds of nanometers or microns could be reached by other mechanisms such as Dexter hopping and coherent exciton motion ${ }^{6,9-10}$ and by a combination of coherent and incoherent exciton motion in the case of $\mathrm{J}$ - and $\mathrm{H}$ aggregates ${ }^{11,35,36}$ ). Within quantum dot films, various methods were developed for the delicate characterization of FRET exciton migration ${ }^{12}$ and yielded diffusion distances of 10-30 
$\mathrm{nm}^{13-14}$. The measured FRET hopping rate in quantum dot films ranged from $30-80 \mathrm{ps}^{15-16}$ to nanoseconds ${ }^{12,16}$ depending on the quantum dot size, separation distance and disorder.

FRET has also been evidenced in films of close-packed semiconductor nanoplatelets (colloidal quantum wells obtained by chemical synthesis ${ }^{17}$ ). It was shown to drastically reduce quantum yield by funneling energy to quenchers ${ }^{18}$ but has also been proposed as a means to promote charge carrier multiplication in photovoltaics by quenching Auger recombination ${ }^{19}$. Several photophysical characteristics of nanoplatelets make them especially prone to FRET: their dipoles present large oscillator strength ${ }^{17}$, their Stokes shift is very low so that their emission and absorption spectra overlap, allowing strong homo-FRET (FRET between similar fluorophores) within a single population of platelets. They have optimal spectral overlap because of their perfect thickness monodispersity and negligible inhomogeneous line width. Moreover, when stacked cofacially, their in-plane dipoles ${ }^{20-21}$ are parallel, their center-tocenter distance (here labelled $\delta$ ) is short, their Förster radius $R_{0}$ is long (estimated to 13.5 $\mathrm{nm}^{5}$ ) and, due to their two-dimensional geometry, the FRET rate decreases as $\left(R_{0} / \delta\right)^{4}$ instead of the usual $\left(R_{0} / \delta\right)^{6}$ dependence ${ }^{5}$.

The extent of FRET interactions between platelets depends however crucially on the ability to stack them with a good order. Self-assembly is a ubiquitous mechanism for nanoparticles ${ }^{22}$. Langmuir protocols have been demonstrated to deposit nanoplatelets with reasonably ordered vertical stacking ${ }^{23-24}$. FRET has been analyzed from the luminescence decays of films of stacked platelets ${ }^{18-19,23,25}$ however the measured FRET rates differed by several orders of magnitude ${ }^{19,25}$, possibly due to the lack of control of disorder ${ }^{5}$. Moreover, in the case of homoFRET within a film of similar platelets, the decay curves provide only very indirect information ${ }^{18}$ so that evidence and quantitative characterization of energy migration by successive homo-FRET jumps remained elusive. Jana et al. have developed the self-assembly of linear chains of nanoplatelets with exceptional order and lengths up to $4 \mu \mathrm{m}$ by an appropriate choice of ligand and solvent ${ }^{26-28}$. The distance between platelets, typically 3 to 6 $\mathrm{nm}$, is highly uniform and set by the length of the ligand molecule. We use here a combination of linear self-assembly of nanoplatelets and microphotoluminescence, which allows us to directly image FRET energy migration. 

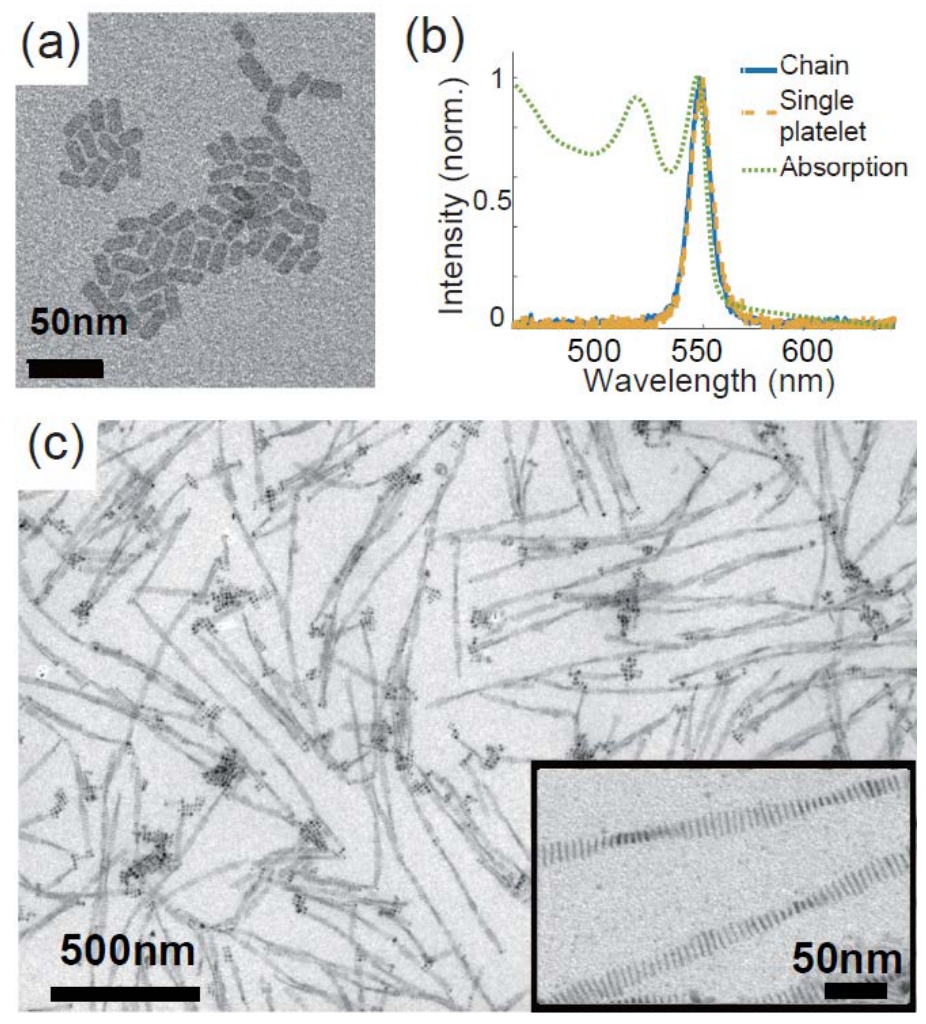

Figure 1 : TEM images of (a) the CdSe nanoplatelets and (c) the nanoplatelets chains. (b) Absorption spectrum (green dotted line) of a nanoplatelets solution. Photoluminescence spectra of a single platelet (yellow dashed line) and a chain (blue full line) under 470-nm excitation.

CdSe nanoplatelets with a 1.5-nm thickness (6 layers of $\mathrm{Cd}$ and 5 layers of Se) were synthesized (protocol in Supporting Information, section A - S.I.-A) corresponding to a fluorescence wavelength of $549 \mathrm{~nm}$. The lateral dimensions were $7 \times 20 \mathrm{~nm}^{2}$ (with 2-nm width dispersion and 4-nm length dispersion) as measured using transmission electron microscopy (TEM) (Fig. 1(a)).The emission spectrum of single platelets (fig. 1(b)) was centered at $549 \mathrm{~nm}$ for all NPLs with negligible dispersion (see fig. S1 in the S.I.). There is no inhomogeneous broadening in such NPLs because the emission wavelength is determined solely by the vertical confinement and the thickness is controlled during the synthesis with atomic layer precision ${ }^{17,37}$. A condition for strong homo-FRET is a good overlap between the absorption and emission spectra : it is the case here (fig. 1(b)) with a Stokes shift of only $2 \mathrm{~nm}$. FRET is also favored if the donor and the acceptor have parallel dipoles. Nanoplatelets are known to behave as an incoherent of two orthogonal in-plane emission dipoles ${ }^{20,21}$ and these dipoles are the same for any excitation polarization (fig. S5) so that two NPLs oriented along parallel planes are expected to always have efficient FRET.

After purification, the assembly takes place in solution during the drying of a dispersion of NPL in the presence of oleic acid. 1D chains of platelets were obtained (Fig. 1(c)) with lengths of 1-2 $\mu \mathrm{m}$ (and a slight helicoidal structure related to the platelets strain-induced twist ${ }^{29-30}$ ). The TEM images show that the distance between the platelets within the stack is very uniform, in 
spite of the helicoidal twist. We estimate from the TEM image a center-to-center distance $\delta$ $=5.7 \mathrm{~nm}$ (see S.I.-A for a discussion of this value). Under fluorescence microscopy observation, the emission spectrum of a NPL chain is identical to the spectrum of a single NPL (fig. 1(b)) : because of the negligible inhomogeneous broadening for NPLs, the redshift signature of hetero-FRET, which has been observed for quantum dots ${ }^{18}$, is not seen here and only pure homo-FRET may occur.

We show on fig. 2(a) the fluorescence image from 3 different chains under wide-field excitation (see S.I.-C for microscopic setup details). Each chain is about 1-2 $\mu \mathrm{m}$ in length, in agreement with electron microscopy images, showing that the spin coating deposition preserved the chain structure. We then changed the excitation source for a pulsed laser positioned at the center of the chain. The typical laser spot was $160 \mathrm{~nm}$ in radius so that if no transfer occurred within the NPL stacks, only this area would have been luminescent. In contrast, we detected, for all the chains considered, fluorescence from an elongated 1- $\mu \mathrm{m}$ to 1.5- $\mu \mathrm{m}$ portion that extended far beyond the spot of the excitation laser (fig. 2(b)).

(a) Wide field excitation
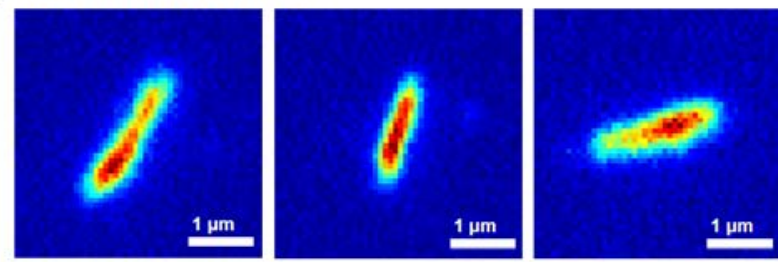

(b) Localized excitation
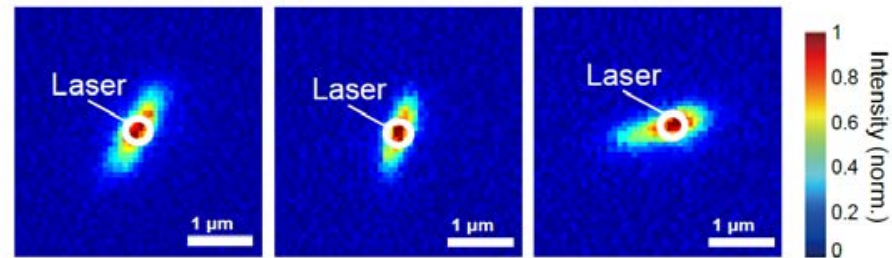

(c)

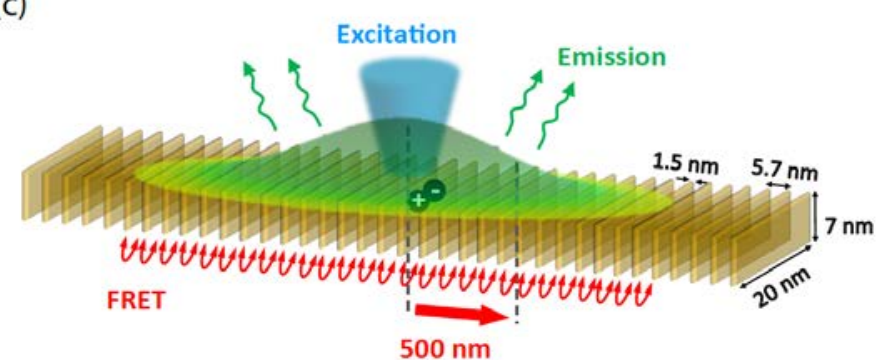

Figure 2 : Images of three platelets chains (a) under wide field excitation and (b) excited locally by a laser spot. (c) Schematic of the proposed FRET migration mechanism.

Part of this elongation can be attributed to the system's response function. The transverse profile $I(y)$ of each chain (fig. 3(b)) can be well fitted by a Gaussian dependence $I(y) \propto$ $e^{-\left(y / l_{y}\right)^{2}}$, with on average a width $l_{y}=196 \mathrm{~nm}$ (fig. 3(c)), in very close agreement to the imaging setup's point spread function (see S.I.-C) of $190 \mathrm{~nm}$. The longitudinal profile $I(x)$ (fig. $3(b))$ is also well fitted by a Gaussian, this time with a width $l_{x}$ ranging between 470 and 720 $\mathrm{nm}$ (fig. 3(c)). The response function of the overall microscopy protocol is, along the chain's axis, a result of the laser spot size (which is plotted in fig. 3(a)) and the imaging point spread 
function and can be described (see S.I.-C) by a Gaussian of width $l_{R F}=246 \mathrm{~nm}$. The response function of the overall microscopy protocol is, along the chain's axis, a result of the laser spot size and the imaging point spread function and can be described (see S.I.-C) by a Gaussian of width $l_{R F}=246 \mathrm{~nm}$. The deconvolved emission profile is thus a Gaussian of width : $l_{F R E T}=$ $\sqrt{l_{x}{ }^{2}-l_{R F}{ }^{2}}$. The distribution of the measured $l_{F R E T}$ values is plotted in fig. $3(\mathrm{~d})$. We find an average length of $500 \mathrm{~nm}$ with a 150-nm dispersion which could be caused by experimental uncertainties as well as by differences between chains such as degree of disorder and twisting.

Because the dielectric index of the chain is higher than the surrounding medium, waveguiding of the excitation beam or of the emitted light might explain our observation. However, finitedifference time domain (FDTD) electromagnetic simulations (see S.I.-D) show that waveguiding effects in our chains are only a few $\%$ of the direct light propagation. We can thus attribute our observations to non-radiative energy transfer between platelets (fig. 2(c)). Two different types of non-radiative hopping mechanisms may occur. FRET is a near-field dipoledipole interaction by which an exciton in one platelet recombines and transfers its energy to a new exciton in the neighbor platelet. Alternatively, Dexter transfer consists in a simultaneous tunneling of the electron and hole to a neighbor platelet ${ }^{5}$. Dexter transfer decays exponentially with distance over a typical 1-nm scale ${ }^{5}$ so that we expect it to be negligible here and attribute our energy transfer to FRET. The FRET migration length of 500 $\mathrm{nm}$ is remarkably long and corresponds to exciton energy diffusion over $l_{F R E T} / \delta \approx 90$ platelets. As compared to literature results presented in the introduction, to our knowledge, self-assembled nanoplatelets thus demonstrate FRET migration lengths larger by more than an order of magnitude with respect to FRET migration in most other system and to any migration reported in quantum dot films.

The migration process and distance may be affected by nonlinear effects such as excitonexciton annihilation (which occurs in semi-conductor nanoparticles through Auger multiexciton recombination). The measurements in figs 2 and 3 were performed under around 5$\mathrm{nW}$ excitation power, within the platelets' linear excitation regime (fig. S8). We estimated that around 0.01 to 0.12 photon was emitted by the NPL chains per laser pulse, so that much less than one exciton was created per laser pulse in the entire chain (see S.I.-E). To further exclude the presence of nonlinear effect, we analyzed the power-dependent fluorescence length on platelet chains (fig. 3(e)) from below 0.1-nW to 100-nW. As a result, the deconvolved emission profile width $l_{F R E T}$ shows no power dependence and is reproducible at low excitation power, ruling out the hypothesis of exciton-exciton annihilation. When the excitation power varies between 0.1 and 10-nW, the decay dynamics of the chain (fig. S9) also remain unchanged, confirming the absence of nonlinear effects. We have also considered cases where the excitation spot was positioned at the end of the NPL chain (fig. S10) : the profile was then asymmetric with no diffusion on one side and around 500-nm diffusion on the other side, in agreement with our other observations. 


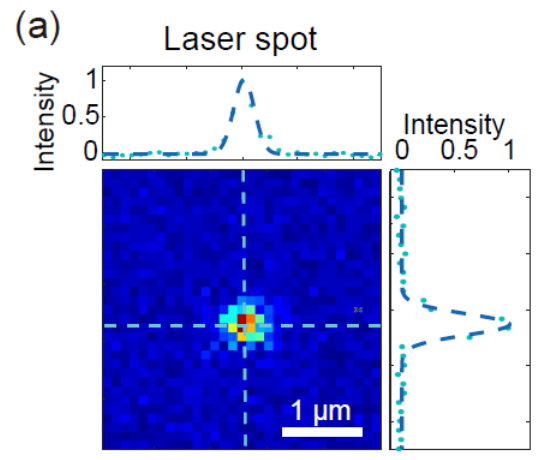

(d)

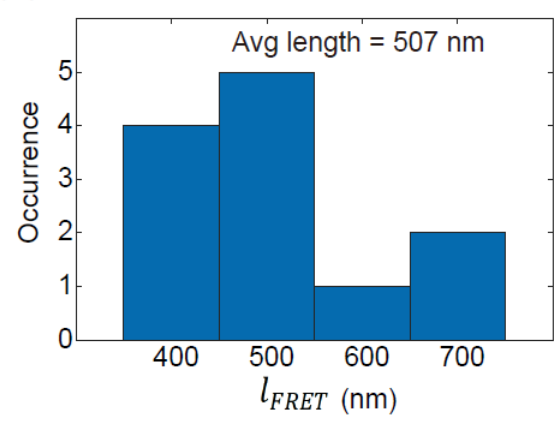

(b)

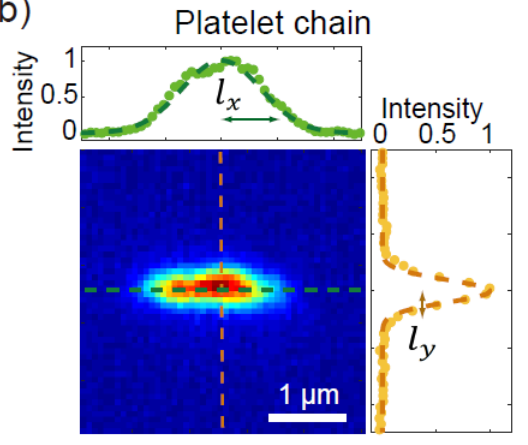

(c)

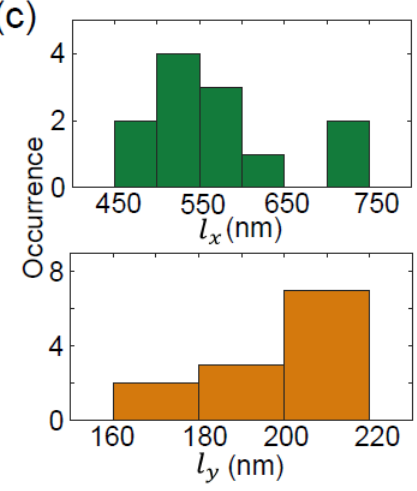

(e)

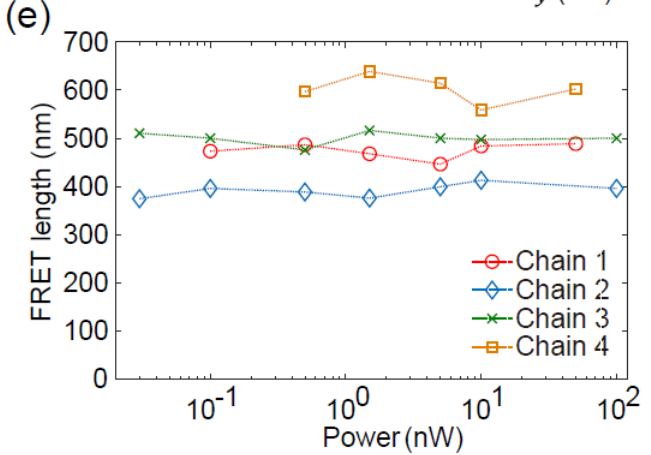

Figure 3 : (a) Map of the excitation laser spot (obtained as described in S.I.-C). (b) Fluorescence image of a typical platelets chain under localized laser 5-nW excitation at its center (dots : experimental profiles; dotted lines: Gaussian fits). (c) Histograms of the fitted $l_{x}$ and $l_{y}$ longitudinal and transverse widths for 12 chains. (d) Histogram of the resolution-corrected migration length values $l_{F R E T}$. (e) Power-dependent FRET length measurements on 4 different chains: excitation powers vary from below 0.1 to $100 \mathrm{nW}$, generating typically $10^{3}-10^{5}$ photon counts/sec.

We now propose a simple model in order to relate the diffusion distance to the transfer rate $\gamma_{t r}$ between neighbor platelets. If an exciton is created at time $t=0$, the probability $n_{i}$ that the $i^{\text {th }}$ platelet is excited may be written ${ }^{18}$ :

$$
\frac{d n_{i}}{d t}=-\left(\gamma_{0}+2 \gamma_{t r}\right) n_{i}+\gamma_{t r}\left(n_{i-1}+n_{i+1}\right)
$$

where the exciton decay rate $\gamma_{0}$ is a sum of respectively radiative and non-radiative decay rates $\gamma_{\text {rad }}$ and $\gamma_{n r}$ (these quantites being assumed the same for all platelets). Over length scales much larger than the center-to-center platelet distance $\delta$, this equation can be rewritten into the diffusion equation ${ }^{6}$ :

$$
\left(\frac{\partial n}{\partial t}\right)(x, t)=-\gamma_{0} n(x, t)+D\left(\frac{\partial^{2} n}{\partial t^{2}}\right)(x, t)
$$

with the diffusion coefficient $D=\delta^{2} \gamma_{t r}$.

A first consequence of this equation (see S.I.-F for demonstration) is that the luminescence decay curve of the whole chain, within this simple model, scales as $e^{-\gamma_{0} t}$, similar to the single- 
platelet decay : FRET is a mechanism of energy diffusion within the chain but not of energy loss for the overall chain. This indicates that the FRET rate cannot be extracted from the measured decay curves (plotted in fig. S4), which is why previous works on the decay of platelet assemblies reported only a theoretical estimate of the FRET rate but no experimental value $^{18,30}$. This illustrates the general difficulty in characterizing exciton diffusion processes, as optical methods provide ambiguous results, while no electrical method can be used because excitons are neutral states ${ }^{12}$.

Our imaging experiment, on the other hand, can provide information on the homo-FRET dynamics within the sample. The image profile is described by

$$
I(x)=\int_{t=0}^{+\infty} \gamma_{r a d} n(x, t) d t
$$

which is shown (see S.I.-F) to decay as

$$
I(x) \propto e^{-|x| / l_{F R E T}}
$$

by introducing the migration distance $l_{F R E T}=\sqrt{D / \gamma_{0}}=\delta \sqrt{\gamma_{t r} / \gamma_{0}}$. This quantity can be interpreted in diffusion terms: the excitation can migrate during a typical time $1 / \gamma_{0}$ before it decays, so that $l_{F R E T}$ is the diffusion length with diffusion coefficient $D$ during a time $1 / \gamma_{0}$.

Within this model, our experimental image profile $I(x)$ should have been an exponential convolved by the apparatus Gaussian response function. However functions of such form could not provide fits as good as the simple Gaussian fit of fig. 3(b). This shows the limits of our simple model and might reveal some degree on diffusion confinement as demonstrated for quantum dot films ${ }^{13}$.

We can however use this model to extract an estimate of the diffusion coefficient and transfer rate from the experimental migration length $l_{F R E T} \approx 500 \mathrm{~nm}$. Given the decay time $1 / \gamma_{0} \approx$ $12 \mathrm{~ns}$ measured on single platelets (fig. S4), we find : $\gamma_{t r}=\gamma_{0}\left(l_{F R E T} / \delta\right)^{2} \sim(1.5 \mathrm{ps})^{-1}$. As a comparison, Förster's equation, which describes the FRET rate between 2 point dipoles, yields in our case (see S.I.-G) a theoretical Förster radius of $17 \mathrm{~nm}$ and a FRET time of $1 / \gamma_{t r}=17 \mathrm{ps}$. Our estimated experimental FRET time of $1.5 \mathrm{ps}$ is an order of magnitude shorter: this probably shows that treating the platelets as points in the Förster radius calculation is a crude approximation because two-dimensional emitters show much higher FRET rates ${ }^{5}$. A description including the electron-hole pair wave functions might be necessary for an appropriate modelling. We note that the transfer is highly efficient over almost one hundred platelets : this suggests that diffusion experiences very little damping by quenchers or stacking faults. It is not clear in our experiment how the diffusion length depends on the position of the excitation spot with respect to quencher sites within the chain. The variability of the quencher positions could induce a dispersion of the measured FRET length, which is not apparent here. Further studies will be necessary in order to analyze to which extent FRET is hampered by defect NPLs.

We can also, from these values, estimate the diffusion coefficient along the chain $D=$ $\gamma_{0} l_{F R E T}^{2} \sim 0.2 \mathrm{~cm}^{2} \mathrm{~s}^{-1}$. This value is much higher than the exciton diffusion coefficients (of the 
order of $10^{-2} \mathrm{~cm}^{2} \mathrm{~s}^{-1}$ at most) reported in organic semiconductors ${ }^{6}$ and quantum dot films $\mathrm{s}^{13,15}$, 31,32 and similar to the estimated diffusion coefficient $\left(0.12 \mathrm{~cm}^{2} \mathrm{~s}^{-1}\right)$ along J-agregate nanotubes ${ }^{36}$. We can also define the efficiency ${ }^{5}$ of neighbor-to-neighbor FRET as $\eta=1-$ $\gamma_{0} / \gamma_{t r}$ and find a value of $99.99 \%$.

As pointed by Rowland et al. ${ }^{19}$, the FRET rate $\left((1.5 \mathrm{ps})^{-1}\right)$ between stacked platelets is much faster than other excitonic mechanisms such as radiative recombination (typical radiative rates of $\left.(12 \mathrm{~ns})^{-1}\right)$, Auger recombination (typical rates of $(150-500 \mathrm{ps})^{-1}$ for $\mathrm{CdSe} / \mathrm{CdS}$ nanoplatelets ${ }^{19}$ ) as well as quenching by trap sites (rate estimated to be $\left.1 /(35 \mathrm{ps})^{18}\right)$. Within a chain of NPLs, we thus expect fast transfer between NPLs to modify the luminescence as compared to isolated NPLs. For instance, collective blinking of clusters of 2 to 10 quantum dots has already been reported due to fast energy transfer between the emitters ${ }^{33}$ (whereas antibunching of clusters of 4-5 quantum dots was attributed to inter-particle Auger interaction ${ }^{34}$ ). The search for even stronger and longer-range FRET-mediated collective photophysical effects between self-assembled chains of nanoplatelets is the topic of our ongoing work.

To conclude, the combined use of fluorescence microscopy and nanoplatelets self-assembly with long range 1D order allowed us to demonstrate Förster energy transfer and diffusion over $500-\mathrm{nm}$ distances. The estimated corresponding FRET rate of $(1.5 \mathrm{ps})^{-1}$ is much faster than other decay mechanisms in platelets so that FRET can be expected to create very strong modifications of the stacked platelets behavior. Self-assembled chains of nanoplatelets constitute a perfect platform for probing these fundamental effects which should have tremendous influence on semiconductor nanoparticles opto-electronics applications.

\section{Supporting information (pdf)}

A - Synthesis and self-assembly protocols, structural chain characterization, B - Single platelet luminescence (spectrum, decay, polarization), C - Imaging setup and resolution, D - Waveguiding simulations, E - Effect of excitation power, F - Diffusion model, G - Theoretical FRET rate, H Excitation at the end of the chain.

\section{Corresponding author}

* laurent.coolen@sorbonne-universite.fr

\section{References}

[1] G. A. Jones and D. S. Bradshaw, Resonance energy transfer : from fundamental theory to recent applications, Front. Phys. 7, 100 (2019),

[2] G. D. Scholes, G. R. Fleming, A. Olaya-Castro and R. van Grondelle, Lessons from nature about solar light harvesting, Nature Chem. 3, 763 (2011),

[3] S. A. Diaz, S. Buckhout-White, M. G. Ancona, C. M. Spillmann, E. R. Goldman, J. S. Melinger and I. L. Medintz, Extending DNA-based molecular photonic wires with homogeneous Förster resonance energy transfer, Adv. Optical Mater. 4, 399 (2016), 
[4] B. L. Cannon, D. L. Kellis, P. H. Davis, J. Lee, W. Kuang, W. L. Hughes, E. Graugnard, B. Yurke and W. B. Knowlton, Excitonic AND logic gates on DNA brick nanobreadboards, ACS Photonics 2, 398 (2015),

[5] B. Guzelturk and H. V. Demir, Near-field energy transfer using nanoemitters for optoelectronics, Adv. Funct. Mater. 26, 8158 (2016),

[6] O. V. Mikhnenko, P. W. M. Blom and T.-Q. Nguyen, Exciton diffusion in organic semiconductors, Energy Environ. Sci. 8, 1867 (2015),

[7] F. Fennel and S. Lochbrunner, Long distance energy transfer in a polymer matrix doped with a perylene dye, Phys. Chem. Chem. Phys. 13, 3527 (2011),

[8] S. M. Menke, W. A. Luhman and R. J. Holmes, Tailored exciton diffusion in organic photovoltaic cells for enhanced power conversion efficiency, Nature Materials 12, 152 (2013),

[9] G. M. Akselrod, P. B. Deotare, N. J. Thompson, J. Lee, W. A. Tisdale, M. A. Baldo, V. M. Menon and V. Bulovic, Visualization of exciton transport in ordered and disordered molecular solids, Nature Comm. 5, 3646 (2014),

[10] F. Dubin, R. Melet, T. Barisien, R. Grousson, L. Legrand, M. Schott and V. Voliotis, Macroscopic coherence of a single exciton state in an organic quantum wire, Nature Phys. 2, 32 (2006),

[11] A. T. Haedler, K. Kreger, A. Issac, B. Wittmann, M. Kivala, N. Hammer, J. Köhler, H.-W. Schmidt and R. Hildner, Nature 523, 196 (2015),

[12] N. Kholmicheva, P. Moroz, H. Eckard, G. Jensen and M. Zambov, Energy transfer in quantum dot solids, ACS Energy Lett. 2, 154 (2017),

[13] G. M. Akselrod, F. Prins, L. V. Poulikakos, E. M. Y. Lee, M. C. Weidman, A. J. Mork, A. P. Willard, V. Bulovic and W. A. Tisdale, Subdiffusive exciton transport in quantum dot solids, Nano Lett. 14, 3556 (2014),

[14] E. M. Y. Lee and W. A. Tisdale, Determination of exciton diffusion length by transient photoluminescence quenching and its application to quantum dot films, J. Phys. Chem. C 119, 9005 (2015),

[15] N. Kholmicheva, P. Moroz, E. Bastola, N. Razgoniaeva, J. Bocanegra, M. Shaughnessy, Z. Porach, D. Khon and M. Zamkov, Mapping the exciton diffusion in semiconductor nanocrystals solids, ACS Nano 9, 2926 (2015),

[16] R. H. Gilmore, E. M. Y. Lee, M. C. Weidman, A. P. Willard and W. A. Tisdale, Charge carrier hopping dynamics in homogeneously broadened PbS quantum dot solids, Nano Lett. 17, 893 (2017),

[17] S. Ithurria, M. D. Tessier, B. Mahler, R. P. S. M. Lobo, B. Dubertret and Al. L. Efros, Colloidal nanoplatelets with two-dimensional electronic structures, Nature Materials 10, 936 (2011),

[18] B. Guzelturk, O. Erdem, M. Olutas, Y. Kelestemur and H. V. Demir, Stacking in colloidal nanoplatelets : tuning excitonic properties, ACS Nano 8, 12524 (2014),

[19] C. E. Rowland, I. Fedin, H. Zhang, S. K. Gray, A. O. Govorov, D. V. Talapin and R. D. Schaller, Nature Materials 14, 484 (2015),

[20] F. Feng, L. T. NGuyen, M. Nasilowski, B. Nadal, B. Dubertret, L. Coolen and A. Maître, Consequence of shape elongation on emission asymmetry for colloidal CdSe/CdS nanoplatelets, Nano Research 11, 3593 (2018), 
[21] F. Feng, L. T. NGuyen, M. Nasilowski, B. Nadal, B. Dubertret, A. Maître and L. Coolen, Probing the fluorescence dipoles of single cubic CdSe/CdS nanoplatelets with vertical or horizontal orientations, ACS Photonics 5, 1994 (2018),

[22] B. Abécassis, Three-dimensional self assembly of semiconducting colloidal nanocrystals : from fundamental forces to collective optical properties, ChemPhysChem 17, 618 (2016),

[23] Y. Gao, M. C. Weidman and W. A. Tisdale, CdSe nanoplatelet films with controlled orientation of their transition dipole moment, Nano Lett. 17, 3837 (2017),

[24] O. Erdem, K. Gungor, B. Guzelturk, I. Tanriover, M. Sak, M. Olutas, D. Dede, Y. Kelestemur and H. V. Demir, Orientation-controlled nonradiative energy transfer to colloidal nanoplatelets : engineering dipole orientation factor, Nano Lett. 19, 4297 (2019),

[25] B. Guzelturk, M. Olutas, S. Delikanli, Y. Kelestemur, O. Erdem and H. V. Demir, Nonradiative energy transfer in colloidal CdSe nanoplatelet films, nanoscale 7, 2545 (2015),

[26] B. Abécassis, M. D. Tessier, P. Davidson and B. Dubertret, Self-assembly of CdSe nanoplatelets into giant micrometer-scale needles emitting polarized light, Nano Lett. 14, 710 (2014),

[27] S. Jana, T. N. T. Phan, C. Bouet, M. D. Tessier, P. Davidson, B. Dubertret and B. Abécassis, Stacking and colloidal stability of CdSe nanoplatelets, Langmuir 31, 10532 (2015),

[28] S. Jana, P. Davidson and B. Abécassis, CdSe nanoplatelets : living polymers, Angew. Chem. Int. Ed. 55, 9371 (2016),

[29] S. Jana, M. de Frutos, P. Davidson and B. Abécassis, Ligand induced twisting of nanoplatelets and their self-assembly into chiral ribbons, Sci. Adv. 3, e:1701483 (2017),

[30] W. D. Kim, D.-E. Yoon, D. Kim, S. Koh, W. K. Bae, W.-S. Chae and D. C. Lee, Stacking of colloidal CdSe nanoplatelets into twisted ribbon superstructures, J. Phys. Chem C 123, 9445 (2019),

[31] E. M. Y. Lee and W. A. Tisdale, Determination of exciton diffusion length by transient photoluminescence quenching and its application to quantum dot films, J. Phys. Chem. C 119, 9005 (2015),

[32] S. J. Yoon, Z. Guo, P. C. dos Santos Claro, E. V. Shevchenko and L. Huang, Direct imaging of longrange exciton transport in quantum dot superlattices by ultrafast microscopy, ACS Nano 10, 7208 (2016),

[33] D. P. Ryan, P. M. Goodwin, C. J. Sheehan, K. J. Whitcomb, M. P. Gelfand and A. Van Orden, Mapping emission from clusters of CdSe/ZnS nanoparticles, J. Phys. Chem. C 122, 4046 (2018),

[34] B. Lv, H. Zhang, L. Wang, C. Zhang, X. Wang, J. Zhang and M. Xiao, Photon antibunching in a cluster of giant CdSe/CdS nanocrystals, Nature Comm. 9, 1536 (2018),

[35] H. Lin, R. Camacho, Y. Tian, T. E. Kaiser, F. Würthner and I. G. Scheblykin, Collective fluorescence blinking in linear J-aggregates assisted by long-distance exciton migration, Nano Lett. 110, 620 (2010),

[36] K. A. Clark, E. L. Krueger and D. A. Vanden Bout, Direct measurement of energy migration in supramolecular carbocyanine dye nanotubes, J. Phys. Chem. Lett. 5, 2274 (2014),

[37] M. D. Tessier, C. Javaux, I. Maksimovic, V. Loriette and B. Dubertret, Spectroscopy of single CdSe nanoplatelets, ACS Nano 6, 6751 (2012). 